\title{
Application of Near-Infrared Spectrometer in Agro-Food Analysis: A Review
}

\author{
Rohinee M. Misal \\ Department of Computer Science \& Information \\ Technology, Dr. Babasaheb Ambedkar \\ Marathwada University Aurangabad (MS), India
}

\author{
Ratnadeep R. Deshmukh \\ Department of Computer Science \& Information \\ Technology, Dr. Babasaheb Ambedkar Marathwada \\ University Aurangabad (MS), India
}

\begin{abstract}
In this review paper applications of Near Infrared (NIR) spectroscopy in agro-food analysis is discussed. NIR spectrometers have a wider application use in agro-food industry and many fields. In agro-food industry application includes pesticide residue detection, chemical detection, microbiological hazard detection, physical hazards detection and food safety and traceability. In addition spectrometer are explored within various fields of agricultural products include food, fruits, vegetables, crops and dairy products, oil and beverages.
\end{abstract}

\section{Keywords}

Spectroscopy, pesticide residue, principal component analysis, artificial neural network

\section{INTRODUCTION}

The agro-food industry is growing widely in the world. Agriculture product and food are important in life and to the economy. It also has very significant role as the population is increasing and increasing demands of consumer for high quality life, quality and safety. NIR spectroscopy applied to the rapid analysis of moisture, protein analysis, and fat content of a wide range of agriculture and food products. It's also widely used in chemistry procedure for qualitative and quantitative analysis in many fields such as food, agriculture, pharmaceuticals, textiles, cosmetics and polymer production [1]. The operation of spectra measurement by spectroscopy is simpler and more convenient than chemistry methods [2]. NIR spectrometer advantages are its non-destructive and in situ analysis. Also include a critical factor for spectrometer development like cost, size, weight, power consumption, robustness, safety, user-friendliness, durability, accuracy of measurement and high performance reliability [3].

Traditional methods and technique Chromatography, HighPerformance Liquid Chromatography, and Gas Chromatography - Mass Chromatography are used for detection and analysis of agro-food. These traditional methods are time consuming, wastage producing, destructive and require the instruments which may contact with hazardous samples. Near-Infrared spectroscopy method is non-destructive, rapid, lower cost, multi parameter results and environmental friendly.

\section{NEAR INFRARED SPECTROSCOPY}

NIR spectroscopy used to determine the Soluble Solid Content (SSC) in apple and many fruits. Also numerous studies have been published on NIR spectroscopy and its application in agro-food industry.

The incident light may reflect, observed or transmitted depending on matter physical structure and chemical composition content. Near-Infrared Radiation ranges from $780 \mathrm{~nm}$ to $2500 \mathrm{~nm}$ of the electromagnetic spectrum. NIR spectrum bands contain hydrogen atom such as $\mathrm{C}-\mathrm{H}, \mathrm{O}-\mathrm{H}, \mathrm{N}-$ $\mathrm{H}, \mathrm{S}-\mathrm{H}$. Organic compounds contain those hydrogen atoms.

Table 1 commonly used Method/Model in NIR Spectroscopy

\begin{tabular}{|c|c|c|c|}
\hline \multirow{11}{*}{$\begin{array}{c}\text { Multivariate } \\
\text { Method }\end{array}$} & \multirow{5}{*}{ Quantitative } & \multirow{3}{*}{$\begin{array}{l}\text { Linear } \\
\text { Methods }\end{array}$} & MLR \\
\hline & & & PCR \\
\hline & & & PLS \\
\hline & & \multirow{2}{*}{$\begin{array}{c}\text { Nonlinear } \\
\text { Method }\end{array}$} & ANN \\
\hline & & & SVM \\
\hline & \multirow{6}{*}{ Qualitative } & \multirow{3}{*}{ Supervised } & SMCA \\
\hline & & & DA \\
\hline & & & \multirow{2}{*}{ KNN } \\
\hline & & \multirow{3}{*}{ Unsupervised } & \\
\hline & & & PCA \\
\hline & & & $\begin{array}{c}\text { Cluster } \\
\text { Analysis }\end{array}$ \\
\hline
\end{tabular}

\section{A. Spectra Information}

Spectral information gets by using a spectrometer. The spectrometer is shown in fig 1 . All information is hidden in the spectra. There are several factors increases the complexity of the spectra like water absorption bands, scattering effects, instrumental noise, etc. Derivative of spectra are a common method used to eliminate baseline from signal spectra.

Table 1 shows the list of commonly used multivariate analysis methods to process NIR spectral information. NIR model accuracy results presented by using Root Mean Square Error of Cross Validation (RMSECV) or Root Mean Square Error of the prediction (RMSEP) as computed using equation 1.

$$
R M S E=\sqrt{\frac{1}{n} \sum_{j=1}^{n}\left(y_{j}-\hat{y}_{j}\right)^{2}}
$$

Where $\hat{y}_{j}$ is the prediction value of the jth observation, yj is the measured value of the observation, and $n$ is the number of the observations.

\section{B. Portable Spectrometer}

Field spec 4 spectrometer (Analytical spectral device, ASD Co. USA) shown in Fig 1 having parameter details in Table 2. Spectrum data export in ASCII text, then it can analyze spectrum data with different software like ASD View Spec Pro. Unscramble and MATLAB. 


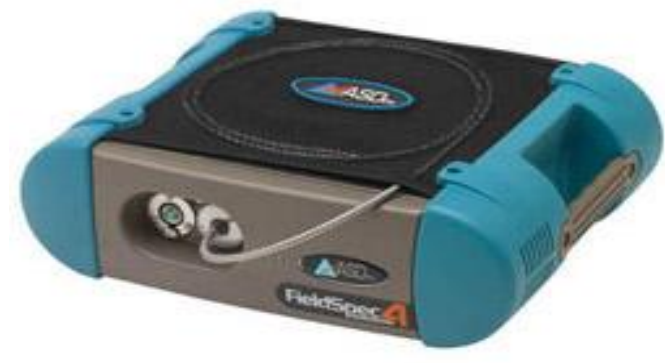

Fig. 1. FieldSpec4 Spectrometer

Table 2 Parameter Details

\begin{tabular}{|c|c|c|}
\hline Table & Specification of field spec 4 spectrometers \\
\hline No. & Performances & Value \\
\hline 1 & Spectral Range (nm) & $350-2500 \mathrm{~nm}$ \\
\hline 2 & Sampling Interval (nm) & $\begin{array}{c}1.4 \mathrm{~nm} @ 350-1000 \mathrm{~nm} \\
1.1 \mathrm{~nm} @ 1000-2500 \mathrm{~nm}\end{array}$ \\
\hline 3 & Spectra Resolution (nm) & $\begin{array}{c}3 \mathrm{~nm} @ 700 \mathrm{~nm}, \\
10 \mathrm{~nm} @ 1400 / 2100 \mathrm{~nm}\end{array}$ \\
\hline 4 & Scanning Time (ms) & $100 \mathrm{~ms}$ \\
\hline
\end{tabular}

\section{APPLICATIONOF SPECTROSCOPY IN AGRO-FOOD INDUSTRY}

- $\quad$ Fruit and Vegetables

NIR spectroscopy is used in the field of fruit and vegetables to detect the SSC (Soluble Solid content), Acidity, Maturity index, sweetness using PH. Fruit quality parameters detection by using spectroscopic measurements.

NIR spectroscopy measures the acidity of Chinese bayberry. The Chines bayberry fruits reflectance measured in NIR 325$1075 \mathrm{~nm}$ range. The logarithms of the reflectance reciprocal (absorbance $(\log 1 / \mathrm{R}))$ of data set were analyzed in order to build the best prediction model for characteristic using several spectral pretreatments and multivariate calibration technique like Partial Least Square regression. The study showed an excellent prediction performance model for prediction of acidity ( $\mathrm{r}=0.963)$, standard error of prediction (SEP) 0.21 with a bias of 0.138 . The NIR technique has an excellent significant greater accuracy for determining the acidity. This NIR non-destructive, fast and accurate technology is used in the food industry that would be beneficial to human health [4].

For instance, an early warning system, based on spectral inputs, would be solved to the enforced reduction of pesticide use. Farmers only have to apply pesticides when abnormalities are detected in the normal growth pattern of the plants. Therefore, it has important implications for biotic stress detection and management in apple orchards [5].

\section{- Chemical Detection}

The main constituents in orange juices are organic acids (citric, tartaric, malic acid, etc.), sugars (fructose, sucrose, etc.), vitamins, and some natural pigments (flavonoids and carotenoids). In the visible region of the electromagnetic spectrum, pigments would produce some influences on the variation of absorbance in the spectra. However, the relationship between $\mathrm{pH}$ and some pigments can provide information to build a PLS calibration about the variation of $\mathrm{pH}$ value in the visible region. Also, the chemical structure of sugars and some organic acids was related to the NIR region [6]. PLS regression as chemo-metrics combined with the VIS-NIRS technique for evaluation of chemical compositions in orange juices is successfully applied by simple models and feature extraction techniques. The PLS regression model was an available alternative for quality detection of orange juices based on VIS-NIRS. Vis/NIR spectroscopy was investigated to predict the soluble solids content (SSC) of rice vinegars based on least squares-support vector machine (LS-SVM) [7]. Vis/NIR spectroscopy combined with LS-SVM regression method was successfully applied for the determination of acetic acid of plum vinegar. The optimal prediction performance was achieved by LSSVM model compared with PLS and MLR models. Vis/NIR spectroscopy combined with chemo-metric method could be applied as an alternative fast way for the determination of acetic acid of plum vinegars. The results may be useful for the process and online monitoring of vinegar fermentation [8].

Industry needs efficient technologies for detection of Pesticide Residue on the surface of fruit in order to maintain its leading position. Nowadays, most of methods to detect the Pesticide Residue are destructive and time-consuming. An efficient, less time consuming and effective Pesticide Residue detection system is urgently needed for the fruit industry to identify the defects rapidly and objectively. The principle components analysis showed that the samples with Pesticide Residue and no Pesticide Residue were perfectly classified by PC1 and PC2. The BP artificial neural network model yielded the corrected prediction as $93 \%$ ratio [9].

- Microbiological

An application by using transmittance spectroscopy to identify the infestation in tart cherry, resulting from present insect activities. The spectra were recorded within a wavelength region between 550 and $980 \mathrm{~nm}$ with a Field Spec Spectroradiometer [10]. The genetic algorithm procedure was used to choose the optimal wavelength regions as a precursor to develop dedicated multispectral vision system for the purpose of detecting internal insect infestation in tart cherries [11]. Approach for detecting internal insect infestation in tart cherries would be limited. According to the GA analysis of the reflectance spectra, visible wavelengths are of less importance than NIR wavelengths for the purpose of distinguishing intact cherries from infested ones. The PLSDA results indicate that models built with three or four GA selected wavelength regions gave a similar classification accuracy of the model built with the full wavelength region, which demonstrates the efficiency of the GA variable selection procedure.

It is possible to use a nondestructive technique to discriminate various fragrant mushrooms, through a hybrid method combining PCA with BP-ANN; a model for the variety discrimination has been established. The Vis/NIR spectral data were first processed by PCA. The first three principal components extracted were then used as the inputs of BPANN. The prediction results were quite encouraging with a correct identification rate over $91 \%$ [12].

Vis/NIRS for determining the $\mathrm{N}$ status of the plant is possible by using a portable spectrophotometer. Vis/NIR spectroscopy and chemo-metrics are used to estimate the $\mathrm{N}$ status of the plant. Chemo-metrics were used as Vis/NIR spectroscopy analysis method to establish models to estimate the $\mathrm{N}$ status of rapeseed and tea plant. The leaf nitrogen accumulation 
almost increased from seedling stage to silking stage, and then decreased from trumpet stage to milky maturity stage. And vegetation indexes and area of red edge of the corn canopy almost increased from seedling stage to trumpet stage, and then decreased from trumpet stage to milky maturity stage. The correlation analysis indicated that the spectral parameters highly correlated with leaf nitrogen accumulation at seeding stage, silking stage and whole growth stage. The correlation relationship of the whole growth cycle was stronger than that of any single growth stage. From a single stage point of view, the seeding stage was the best growth period for monitoring the leaf nitrogen accumulation. The regression analysis displayed that the all regression models between spectral $\mathrm{s}$ and leaf nitrogen accumulation were all significant at the whole growth stage. The research demonstrated that it is a feasible method for quantificational evaluation of corn leaf nitrogen accumulation by hyper-spectral remote sensing.

\section{CONCLUSION}

The NIR Spectroscopy is useful in a wide number of applications in the agro-food industry. Advantages of NIR Spectroscopy: Non-destructive analyses, Multi-parameter, lower costs, in situ, real time and environmental friendliness. Nevertheless, the technique has drawbacks: requiring not only an accurate reference analysis, but also a large and updated calibration data set. Therefore, NIR spectroscopy and imaging technique is very useful for qualitative analysis to evaluate whether food is safe or not. In order to improve the accuracy of quantitative analysis for low concentration hazards in food samples, special sample pretreatment methods are adopted sometimes, such as silica gel used as a sorbent for enriching the object's concentration of a contaminant.

\section{ACKNOWLEDGMENT}

This work is supported by the Department of Science and Technology, New Delhi, under the FIST (Funds for Improvement of Science and Technology Infrastructure) program, sanctioned to Department of Computer Science and Information Technology, Dr. Babasaheb Ambedkar Marathwada University, Aurangabad-431004, (MS), India.

\section{REFERENCES}

[1] S. Gunasekaran, J. Irudayaraj. "Optical Methods: Visible, NIR, and FTIR Spectroscopy". In: S. Gunasekaran, editor. Nondestructive Food Evaluation: Techniques to Analyze Properties and Quality. New York: Marcel Dekker, 2001. Pp. 1-37

[2] Muik, B., Lendl, B., Molina-D’1az, A., Ortega-Caldern, D., and Ayora-Canada, M.J, "Discrimination of olives according to fruit quality using Fourier transform Raman spectroscopy and pattern recognition techniques." J. Agr. Food Chem., 52: 6055-6060.

[3] Y. L. Yan, L. L. Zhao, D. H. Han, and S. M. Yang, The Foundation and Application of Near Infrared
Spectroscopy

Analysis. China Light Industry Press, Beijing, 2005.

[4] Kexin Xu; Qingming Luo; Da Xing; Alexander V. Priezzhev; Valery V. Tuchin Tianjin, "Nondestructive measurement of acidity of Chinese bayberry using Vis/NIR spectroscopy techniques", Fourth International Conference on Photonics and Imaging in Biology and Medicine, 60472D (October 27, 2006); doi:10.1117/12.710950

[5] Stephanie Delalieux, Jan van Aardt, Wannes Keulemans, and Pol Coppin, "Detection of biotic stress (venturia inaequalis) in appleTrees using hyperspectral analysis", EARSeL and Warsaw University, Warsaw 2005.

[6] Haiyan Cen, Yong He, And Min Huang, "Measurement of Soluble Solids Contents and $\mathrm{pH}$ in Orange Juice Using Chemometrics and Vis-NIRS", J. Agric. Food Chem, 2006, 54, 7437-7443

[7] Fei Liu, Yong He, Li Wang, "Application of Least Squares-Support Vector Machine for Measurement of Soluble Solids Content of Rice Vinegars Using Vis/NIR Spectroscopy", International Conference on Computational Intelligence and Security, 2007, IEEE, DOI 10.1109/CIS.2007.212

[8] Zunyi Wang, Fei Liu, Yong He, "Comparison and Determination of Acetic Acid of Plum Vinegar Using Visible/Near Infrared Spectroscopy and Multivariate Calibration", World Congress on Computer Science and Information Engineering, 2008 IEEE, DOI 10.1109/CSIE. 728

[9] Dai Fen, Hong Tiansheng, Zhang Kun, Hong Ya, "Nondestructive Detection of Pesticide Residue on Longan Surface Based on Near Infrared Spectroscopy", International Conference on Intelligent Computation Technology and Automation, 2010, IEEE, DOI 10.1109/ICICTA.2010.477

[10] Juan Xing, Daniel Guyer, "Detecting internal insect infestation in tart cherry using transmittance spectroscopy", 2008, Elsevier, doi:10.1016/j.postharvbio.2008.03.018

[11] Juan Xing. Daniel Guyer, Diwan Ariana, Renfu Lu, "Determining optimal wavebands using genetic algorithm for detection of internal insect infestation in tart cherry", Sens. \& Instrumen. Food Qual. (2008) 2:161-167 DOI 10.1007/s11694-008-9047-z

[12] Haiqing Yang, Yong He, "Nondestructive Variety Discrimination of Fragrant Mushrooms Based on Vis/NIR Spectral Analysis", 2008 IEEE DOI 10.1109/CISP.2008.627 